\title{
STUDY OF THE INTERACTIONS BETWEEN PENICILLIUM OXALICUM CURRIE \& THOM AND ALTERNARIA ALTERNATA (FR.) KEISSLER
}

\author{
F. Sempere*, M.P Santamarina
}

Departamento de Ecosistemas Agroforestales. Escuela Técnica Superior del Medio Rural y Enología. Universidad Politécnica de Valencia. Avda. Blasco Ibañez, 21. 46010-Valencia. España.

Submitted: July 21, 2009; Returned to authors for corrections: December 02, 2009; Approved: February 18, 2010.

\begin{abstract}
The aim of this research was the analysis of the possible antagonistic effect of Penicillium oxalicum over the pathogen rice fungus $A$. alternata under different conditions of temperature, water activity and culture media. The macroscopic study of the dual growth revealed that according to the Index of Dominance $P$. oxalicum was more competitive that A. alternata at $25^{\circ} \mathrm{C}$ whereas at $15^{\circ} \mathrm{C}$ was this species. Microscopic analysis showed that $P$. oxalicum was a mycoparasite of A. alternata at all conditions tested. The antagonist penetrated into A. alternata and disintegrated its conidiophores and conidia. The results suggests that $P$. oxalicum may be a possible biological control agent of the rice pathogens in a future.
\end{abstract}

Key words: Alternaria alternata, Penicillium oxalicum, rice, water activity, mycoparasitism.

\section{INTRODUCTION}

Alternaria alternata (Fr.) Keissler is a pathogen fungus of several crops causing losses of different consideration. Also this species is known to produce several mycotoxins in foods: alternariol, alternariol monomethyl ether, etc.

Rice grains are frequently contaminated with various Alternaria species, particularly A. alternata and its presence has been reported in different worldwide areas $(1,12,13,18)$. In this cereal the strain produces a reduction of the initial quality: undesirable caryopsides' colorations, brittle grains, weight loss, reduction of nutritional constituents, etc.

Application of chemical fungicides is a common management strategy to combat the pathogens on rice $(5,20)$. But it is essential the application the respectful alternatives with the environment. The success of biological control agents for the control of others crops pathogens fungi require researches of new organisms for a possible future control of these strains on the different production phases. Few studies have been carried for the determination of the biological control agents of rice fungi. Currently no available biocontrol agents of these important cereal pathogens exist for its commerzialitation.

The aim of this research was the analysis of the possible antagonistic effect of Penicillium oxalicum over A. alternata under different conditions of temperature and water activity. The experiment was undertaken macroscopically and microscopically using both light microscopy (LM) and cryoscanning electron microscopy (cryo-SEM).

\section{MATERIALS AND METHODS}

\section{Microscopic fungi}

The fungus Alternaria alternata was isolated from samples of rice grains collected from different rice fields and cooperatives of the main rice producing areas in Valencia. 
The fungus Penicillium oxalicum was obtained of the fungal collection of the Department of Agroforest Ecosystems in the School of the Rural Environment and Enology.

\section{Substrates}

The substrates used in this study were Rice Extract Agar and rice of Valencia. The synthetic medium Rice Extract Agar was obtained from grains of rice. The water activity $\left(a_{\mathrm{w}}\right)$ of this basal medium was modified by the addition of different amounts of glycerol to obtain five different levels of water activities $(0.995,0.98,0.95,0.90$ and 0.85$)$.

The sterilized rice grains with a solution of sodium hypochlorite were deposited 48 hours in the different solutions for set the water activity $\left(0.995,0.98\right.$ and $0.95 a_{\mathrm{w}}: 2.5,11$ and $23.5 \mathrm{~g}$ glycerol / $100 \mathrm{~mL}$ distilled water) (15).

\section{Ecophisiological study}

The ecophisiological study of the two fungi was carried in Rice Extract Agar at the different water activities. Eight millimetres diameter disks of Penicillium oxalicum and Alternaria alternata, $45 \mathrm{~mm}$ apart, obtained from the growing margins of the fungus colonies grown in PDA at $25^{\circ} \mathrm{C}$ for 5 days were inoculated in the Petri Plates. The samples were incubated at two temperatures $\left(15^{\circ} \mathrm{C}\right.$ and $\left.25^{\circ} \mathrm{C}\right)$.

In total 10 treatments were performed combining five water activities $(0.85,0.90,0.95,0.98$ and 0.995$)$ and two temperatures $\left(15\right.$ and $\left.25{ }^{\circ} \mathrm{C}\right)$.Each treatment was repeated four times.

To maintain the water activity during the testing period, Petri plates of the same value were placed in polyethylene boxes containing solutions with the corresponding water activity value. Water activity was checked using an Aqualab (Decago, Inc., Pullman, WA, USA).

Daily the diameters of the growing colonies were measured in two directions at right angles to each other during a period of five days. A linear regression of increase in radius (in $\mathrm{mm}$ ) against time (in days) was used to obtain the growth rates $\left(\mathrm{mm} \mathrm{day}^{-1}\right)$ for each set of treatment conditions. The computer software used was Microsoft Excel 2003.
The analysis of variance (ANOVA) with significance values of $\mathrm{P}<0.01$ was used to determine the influence of parameters water activity $\left(a_{\mathrm{w}}\right)$, temperature $(\mathrm{T})$, species $(\mathrm{E})$ and their interactions $\left(a_{\mathrm{w}} \times \mathrm{T}\right)\left(a_{\mathrm{w}} \times \mathrm{E}\right)(\mathrm{T} \times \mathrm{E})$ on dual fungal growth rates. STATGRAPHICS Plus 5.0 software (Stat Point, Inc., Herndon, Virginia, USA) was used in the study.

\section{Macroscopic study of the interaction}

Rice Extract Agar at three water activities (0.995, 0.98 and 0.95) was the substratum used for the macroscopic study of the interaction. After the ecophisiological study the Petri plates were incubated a period of eight weeks for the determination of the interactions.

According to method proposed by Magan and Lacey (9), the interactions were determined and numerical scores were assigned for $P$. oxalicum and A. alternata with the objective to obtain the Index of Dominance at the water activities and temperatures assayed. Mutual intermingling [1]; mutual antagonism on contact or with free space between fungus colonies < $2 \mathrm{~mm}$ [2]; mutual antagonism at a distance [3]; dominance on contact [4for the dominant species, 0 for the inhibited species]; dominance at a distance [5 for the dominant species, 0 for the inhibited species].

\section{Microscopy study of the fungi relations}

A scanning microscope JEOL JSM 5410 and a light microscope Olympus PM-10AK3 were used in this study. Microscopic analysis was performed on Rice Extract Agar and full rice grains. The water activities experimented were 0.95, 0.98 and 0.995 at both temperatures

Previously the preparation of the samples for its microscopical observation was described. For the analysis in the synthetic medium, both fungal species were inoculated in REA squares at different $a_{\mathrm{w}}$ levels $5 \mathrm{~mm}$ apart, mounted on glass slides in glass rods inside Petri plates of $90 \mathrm{~mm}$ under conditions of total asepsis. Coverslips were placed on the films. To maintain the levels of water activity, filter paper disks impregnated with different solutions $(0.995,0.98$ and 0.95 aw: 2.5, 11 and $23.5 \mathrm{~g}$ glycerol/100 $\mathrm{ml}$ distilled water) were 
aseptically placed on the Petri plates. For the analysis of fungal interaction in rice grains, first the samples were sterilized with a solution of sodium hypochlorite and were deposited $48 \mathrm{~h}$ in the solutions described above for setting the water activities. The study was conducted in the same conditions that the analysis in the synthetic medium, except for the strains that were inoculated $3 \mathrm{~mm}$ apart. For cryo-scanning electron analysis no coverslips were deposited on the substrates (16).

The plates with the dual microculture in Rice Extract Agar and rice grains were incubated a period between 7-30 days depending on both temperature and water activity.

\section{RESULTS}

\section{Growth at different environmental conditions}

Figure 1 shows the growth rates of Penicillium oxalicum and Alternaria alternata at different $a_{\mathrm{w}}$ levels and temperatures. Maximum growth rate in both fungal species occurred at a temperature of $25^{\circ} \mathrm{C}$ and water activities of 0.995 for A. alternata and 0.98 for P. oxalicum, with values of 6.18 $\mathrm{mm} \mathrm{day}{ }^{-1}$ and of $4.16 \mathrm{~mm} \mathrm{day}^{-1}$ respectively.

At $0.995 a_{\mathrm{w}}$ A. alternata exhibited higher growth rates that P. oxalicum. Minimal differences between the growth rates of both isolates at the others water activities and both temperatures were observed. For example at 0.98 and $25^{\circ} \mathrm{C}$, $4.75 \mathrm{~mm}$ day $^{-1}$ was the numerical value registered for $A$. alternata and $4.16 \mathrm{~mm} \mathrm{day}^{-1}$ for $P$. oxalicum.

The minimum amount of water activity producing growth in Rice Extract Agar occurred at $0.90 a_{\mathrm{w}}$ for A. alternata and at $0.85 a_{\mathrm{w}}$ for P. oxallicum. Although initially development was not registered in these conditions, by the end of the eight weeks it was observed.
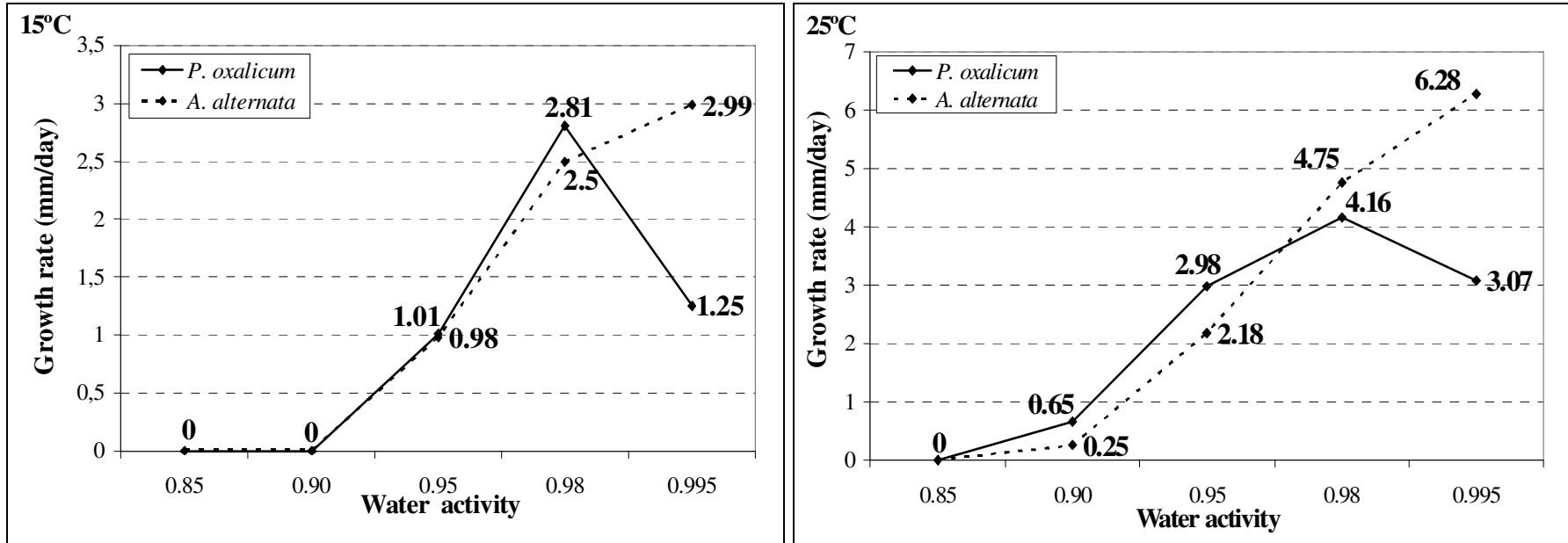

Figure 1. Ecophisiological study of Penicillium oxalicum and Alternaria alternata dual culture in Rice Extract Agar at different temperatures and water activities.

Dual growth of $P$. oxalicum and A. alternata was significantly affected by the simple factors: water activity, temperature, species and the interactions $\left(a_{\mathrm{w}} \mathrm{x} \mathrm{T}, a_{\mathrm{w}} \mathrm{xE}\right)(\mathrm{P}$ $<0.01)$. The dual factor TxE had a significant effect $(\mathrm{P}<0.05)$ (Table 1).

Initially the inoculation of both isolates in the same substrate not affected their growth. Later the colonies came into contact, the interaction significantly influenced their development.

\section{Interactions according to the method of Magan and Lacey}

When $P$. oxalicum and A. alternata were inoculated on Rice Extract Agar, three type of interactions were registered at the different conditions experimented (Figure 2). At $0.98 a_{\mathrm{w}}$ 
and both temperatures $A$. alternata inhibited $P$. oxalicum on contact. Both species continued growing until both colonies came into contact. Later, A. alternata grew over P. oxalicum. The same type of interaction was registered at $0.995 a_{\mathrm{w}}$ and $15^{\circ} \mathrm{C}$.

Mutual antagonism on contact of both isolates at $0.95 a_{\mathrm{w}}$ and $15^{\circ} \mathrm{C}$ was observed. At the rest of conditions $P$. oxalicum inhibited A. alternata on contact.

Table 1. Anaysis of variance of the growth rate of $P$. oxalicum and A. alternata; significance of water activity $\left(a_{\mathrm{w}}\right)$, temperature (T), species (E) and their interaction. (DF) Degrees of freedom. (MS) Mean squares. **Indicates that the factor elicited a significant effect $(\mathrm{P}<0.01)$. *Indicates that the factor elicited a significant effect $(\mathrm{P}<0.05)$.

\begin{tabular}{lllcl}
\hline FACTOR & DF & MS & F-ratio & P-value \\
\hline$a_{\mathrm{w}}$ & 4 & 1636.63 & 115.39 & $0.0000^{* *}$ \\
$\mathrm{~T}$ & 1 & 2263.38 & 159.58 & $0.0000^{* *}$ \\
$\mathrm{E}$ & 1 & 332.151 & 23.42 & $0.0000^{* *}$ \\
$a_{\mathrm{w}} \mathrm{x}$ T & 4 & 358.826 & 25.30 & $0.0000^{* *}$ \\
$a_{\mathrm{w}} \mathrm{x}$ E & 4 & 233.133 & 16.44 & $0.0000^{* *}$ \\
$\mathrm{TxE}$ & 1 & 52.2006 & 3.68 & $0.0558^{*}$ \\
\hline
\end{tabular}

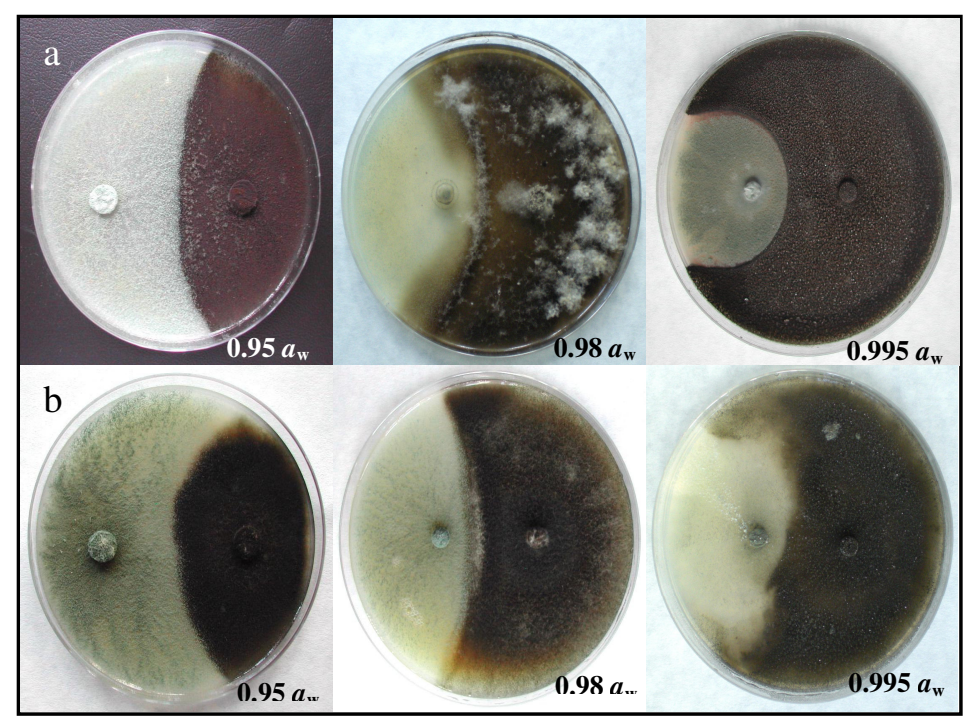

Figure 2. Dual cultures between $P$. oxalicum and A. alternata after 8 weeks on Rice Extract Agar at different temperatures and water activities. Row a: $25^{\circ} \mathrm{C}$. Row b: $15^{\circ} \mathrm{C}$. Left: P. oxalicum. Right: A. alternata.
According to the Index of Dominance, $P$. oxalicum was a species more competitive that A. alternata at $25^{\circ} \mathrm{C}$ (Table 2).

Macroscopically, in all abiotic factors tested, no apparent changes of $P$. oxalicum and A. alternata colonies were observed. Cultural characters appeared similar to that of the fungi in singles cultures at the different temperatures and water activities.

Table 2. Index of Dominance $\left(\mathrm{I}_{\mathrm{D}}\right)$. $\mathrm{I}_{\mathrm{D}}$ refers to sum of scores at $25^{\circ} \mathrm{C}$ and $15^{\circ} \mathrm{C}$ for $P$. oxalicum competing with A. alternata based on the interaction scores for each species. Dominance on contact (4 for the dominant species, 0 for the inhibited species). (X) Analysis of interaction was discarded.

\begin{tabular}{clcccccc}
\hline Temperature & Fungus & $\mathbf{0 . 9 9 5}$ & $\mathbf{0 . 9 8}$ & $\mathbf{0 . 9 5}$ & $\mathbf{0 . 9 0}$ & $\mathbf{I}_{\mathbf{D}}$ \\
& species & $\boldsymbol{a}_{\mathrm{w}}$ & $\boldsymbol{a}_{\mathrm{w}}$ & $\boldsymbol{a}_{\mathrm{w}}$ & $\boldsymbol{a}_{\mathbf{w}}$ & \\
\hline $25{ }^{\circ} \mathrm{C}$ & P. oxalicum & 4 & 0 & 4 & $\mathrm{X}$ & 8 \\
\cline { 2 - 7 } & A. alternata & 0 & 4 & 0 & $\mathrm{X}$ & 4 \\
\hline \multirow{2}{*}{$15^{\circ} \mathrm{C}$} & P. oxalicum & 0 & 0 & 2 & $\mathrm{X}$ & 2 \\
\cline { 2 - 7 } & A. alternata & 4 & 4 & 2 & $\mathrm{X}$ & 10 \\
\hline
\end{tabular}

\section{Efficacy of $P$. oxalicum isolate against $A$. alternata on different substrates}

Microscopic analysis of the interactions on Rice Extract Agar and full rice grains revealed that $P$. oxalicum was a mycoparasite of A. alternata at all conditions of temperatures and water activities.

Alternaria alternata sporulated at the same environmental conditions that when this fungus were inoculated individually (0.995 $a_{\mathrm{w}}$ to $0.95 a_{\mathrm{w}}$ at both temperatures), but $P$. oxaliucm parasited the different reproductive structures of the pathogen when the isolates were grown dually. This rice pathogen species in REA medium and full rice grains presented septate hyphae and pluricellular conidia with transverse and, in some cases, longitudinal septa irregularly distributed, ovoid, pyriform, ellipsoidal or oval-shaped, brownish in colour, with rough surface ornamentation, which were born from simple walled conidiophores with smooth walls, or from the previous spore, giving rise in this case to a chain that tends to get 
branched if the spore generates more than one sprout.

Penicillium oxalicum in both substrates jointly $A$. alternata and individually sporulated at all temperatures and temperatures assayed $\left(0.995 a_{\mathrm{w}}\right.$ to $0.85 a_{\mathrm{w}}$ at 15 and $\left.25^{\circ}\right)$. Terverticillate Penicillium with smooth conidiophores (stipe, metulae, branches and phialides), ampulliformes to cylindrical phialides with a conical apical portion. Spherical to ellipsoidal conidia, unicellular and hyaline. Smooth to slightly rough cell wall conidia in no branched long chains originated from the phialides.

In the figures $3 \mathrm{~A}, 3 \mathrm{~B}, 3 \mathrm{C}, 4 \mathrm{~A}, 4 \mathrm{~B}$ and $4 \mathrm{C}$ it can be seen the initial contact and recognition between the mycoparasite $P$. oxalicum and its host A. alternata. Later, $P$. oxalicum penetrated into A. alternata and disintegrated its conidiophores and conidia (Figures 3D and 4D).

The attack of the A. alternata hypha not were observed in the study at the different conditions.

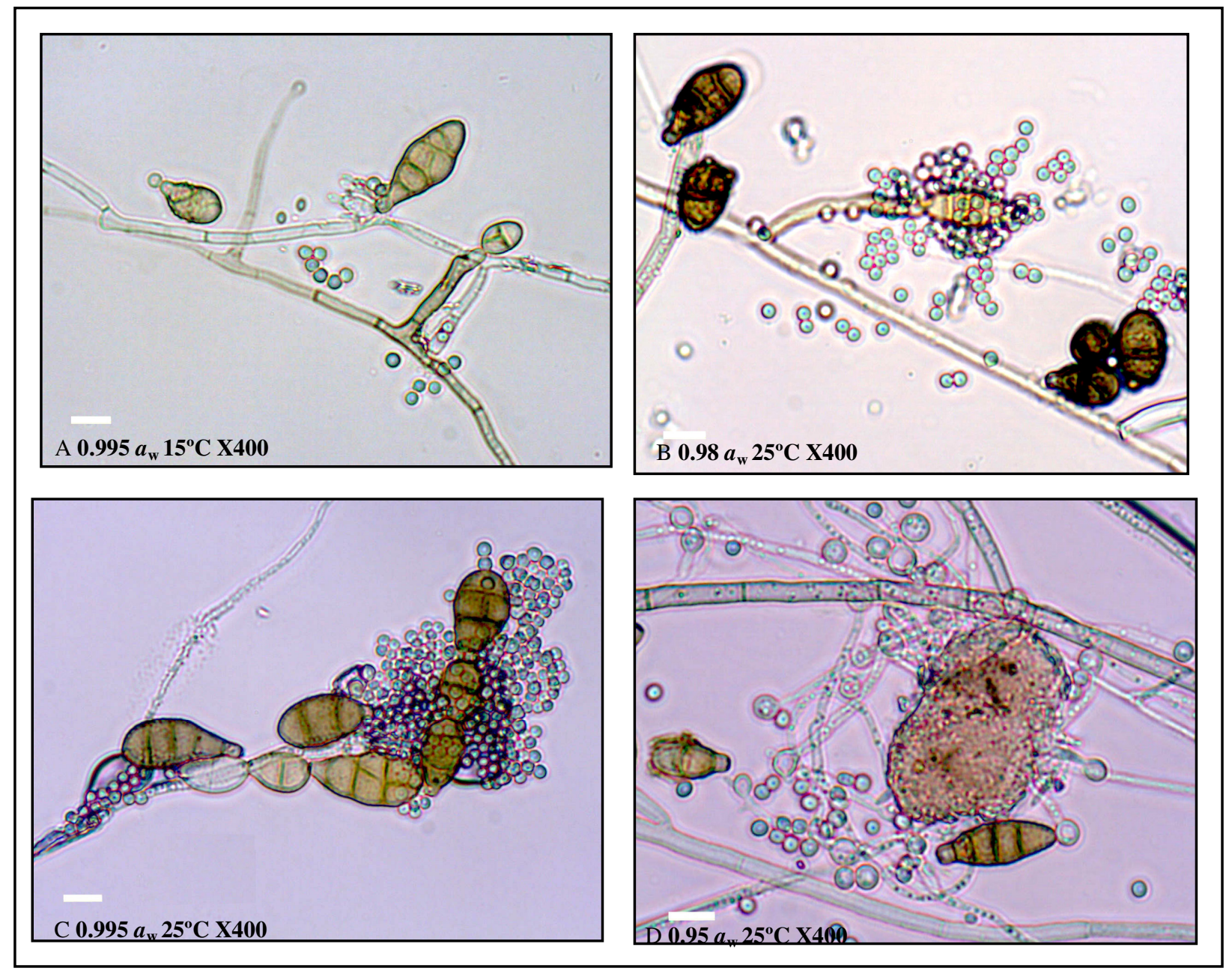

Figure 3. Light micrographs of the dual microculture of $P$. oxalicum and A. alternata at different temperatures and water activities. A: Physical examination and contact between $P$. oxalicum and the conidiophores and conidia of A. alternata. B, C: Antagonist attack on the conidia of A. alternata. D: Completely disintegrated conidia of A. alternata by the action of $P$. oxalicum. $\approx \approx \mu \mathrm{m}$. 


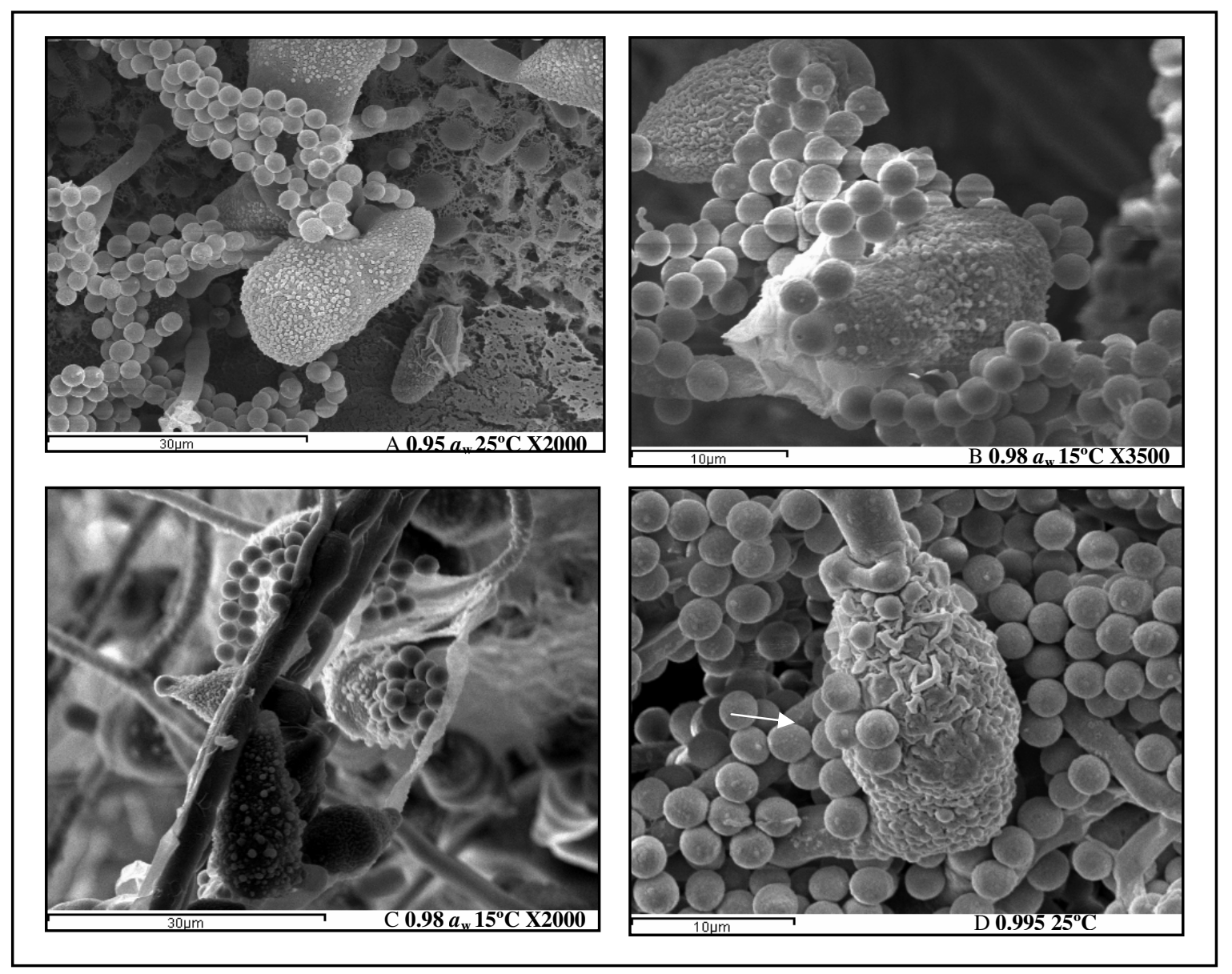

Figure 4. Cryo-scanning electron micrographs showing mycoparasitism of A. alternata by P. oxalicum at different temperatures and water activities. A, B, C: Initial contact, attack and penetration of P. oxalicum into A. alternata. D: Disintegrating conidium of A. alternata by the action of $P$. oxalicum. It can be seen the conidia of $P$. oxalicum coming out of a conidium of $A$. alternata (see arrow). A, B: The fungi were inoculated on Rice Extract Agar. C, D: Full rice grain.

\section{DISCUSSION}

This study is the first detailed the mycoparasitism of $P$. oxalicum over A. alternata. The analysis performed in this work reveals that the mechanisms by which $P$. oxalicum antagonize A. alternata vary depending on the conditions of temperature and water activity. At $0.995 a_{\mathrm{w}} 25^{\circ} \mathrm{C}$ and at 0.95 $a_{\mathrm{w}}$ and both temperatures $P$. oxalicum exerted synergistically two mechanisms of antagonism: mycoparasitism and competition for space and nutrients.

Previously Trichoderma species has been tested over $A$. alternata. Trichoderma isolates antagonized A. alternarta through different mechanisms such as competition for space and nutrients, mycoparasitism and possible antibiosis $(10,11$, 16).

Different researches described the antagonistic mechanism of induction of resistance for Penicillium oxalicum. In this sense, Penicillium oxalicum is a promising fungal agent for biological control of tomato diseases, such as those caused by Fusarium oxysporum f. sp. lycopersici (2, 3), Verticillium dahliae $(6,7,14)$. Also this antagonistic agent has been tested over strawberry and pea fungal strains and others crop pathogens with good results $(4,19)$.

When this species was confronted with other species belonging to the dominant mycobiota of Valencia rice: Nigrospora oryzae, $P$. oxalicum antagonized this rice pathogen 
by mycoparasitism and competition for space and nutrients in all conditions assayed (17).

The degradation and penetration of cell walls of $A$. alternata conidiophores suggests that $P$. oxalicum produce antifungal components and extracellular metabolites like cell wall degrading enzymes chitinases, glucanases and proteases. Ma et al. (8) reported the potential of Penicillium striatisporum, as a biological control agent of soil-borne diseases caused by plant pathogens such as Phytophthora spp., Cladosporium cucumerium, and Sclerotinia sclerotiorium and the authors suggested that the suppression of these pathogens may be due to the production of toxic metabolites by this biological control agent. In this study no mycoparasitism was observed.

Alternaria alternata species can contaminate rice from its cultivation to harvest, during its transportation and storage, and in various production phases causing loss of different consideration. The results of this study are the first to describe the parasitism of A. alternata by $P$. oxalicum in rice grains and Rice Extract Agar in vitro conditions. P. oxalicum may be a good biological control agent of the rice pathogens fungi in a future but more researches is necessary for this consideration.

\section{REFERENCES}

1. Broggi, L.E.; González, H.H.L.; Resnik, S.L.; Pacin. (2007). A. alternata prevalence in cereal grains and soybean seeds from Entre Ríos, Argentina. Rev. Iberoam. Micol. 24, 47-51.

2. De Cal, A.; Melgarejo, P. (2001). Repeated applications of Penicillium oxalicum prolongs biocontrol of fusarium wilt of tomato plants. Eur. J. Plant Pathol. 107, 805-811.

3. De Cal, A.; Pascual, S.; Melgarejo, P. (1995). Biological control of Fusarium oxysporum f. sp. lycopersici. Plant Pathol. 44, 909-914.

4. De Cal, A.; Redondo, C.; Sztejnberg, A.; Melgarejo, P. (2008). Biocontrol of powdery mildew by Penicillium oxalicum in open-field nurseries of strawberries. Biol. Control 47, 103-107.

5. Groth, D.E. (2008). Effects of cultivar resistance and single fungicide application on rice sheath blight, yield, and quality. Crop Prot. 27, 1125-1130.

6. Larena, I.; De Cal, A.; García-Lepe, R.; Melgarejo, P. (2001). Biocontrol of tomato diseases by Penicillium oxalicum. In: Dehne, H.-
W., Gisi, U., Kuck, K.H., Russell, P.E., Lyr, H. (Eds.), Modern Fungicides and Antifungal Compunds III. AgroConcept Gmbh, Bonn, pp. 387-394.

7. Larena, I.; Sabuquillo, P.; Melgarejo, P.; De Cal, A. (2003). Biocontrol of Fusarium and Verticillium wilt of tomato by Penicillium oxalicum under greenhouse and field conditions. J. Phytopathol. 151, 507-512.

8. Ma, Y.; Chang, Z; Zhao, J., Zhou M. (2008) Antifungal activity of Penicillium striatisporum Pst10 and its biocontrol effect on Phytophthora root rot of chilli pepper. Biol. Control 44, 24-31.

9. Magan, N.; Lacey, J. (1984). The effect of water activity, temperature and substrate on interactions between field and storage fungi. Trans. Brit. Mycol. Soc. 82, 83-93.

10. Mónaco, C.; Cisterna, M.; Perelló, A.; Dal Bello, G. (2004). Preliminary studies on biological control of the blackpoint complex of wheat in Argentina. World J. Microbiol. Biotechnol. 20, 285-290.

11. Nallathambi, P.; Umamaheswari, C.; Thakore, B.B.L.; More, T.A. (2009). Post-harvest management of ber (Ziziphus mauritiana Lamk) fruit rot (Alternaria alternata Fr. Keissler) using Trichoderma species, fungicides and their combinations. Crop Prot., Article in press.

12. Piñeiro, F.; García, J. (2000). La sanidad en el arrozal valenciano. Enfermedades. Vida Rural 108, 26-30.

13. Reddy, K.R.N.; Reddy, C.S.; Muralidharan, K. (2005). Characterization of aflatoxin B1 produced by Aspergillus flavus isolated from discolored rice grains. J. Mycol. Plant Pathol. 35(3), 470-474.

14. Sabuquillo, P.; De Cal, A.; Melgarejo, P. (2006). Biocontrol of tomato wilt by Penicillium oxalicum in different crop conditions. Biol. Control $37,256-265$.

15. Sempere, F.; Santamarina, M.P. (2006). Microscopic and macroscopic study of the interaction between Alternaria alternata (Fr.) Keissler and Nigrospora oryzae (Berk. \& Broome) Petch. Ann. Microbiol. 56, 101107.

16. Sempere, F.; Santamarina, M.P. (2007). In vitro biocontrol analysis of Alternaria alternata (Fr.) Keissler under different environmental conditions. Mycopathologia 163, 183-190.

17. Sempere, F.; Santamarina, M.P. (2008). Suppression of Nigrospora oryzae (Berk. \& Broome) Petch by an aggressive mycoparasite and competitor, Penicillium oxalicum Currie \& Thom. Int. J. Food Microbiol. 122, 35-43.

18. Tonon, S.A.; Marucci, R.S.; Jerke, G.; Garcia, A. (1997). Mycoflora of paddy and milled rice produced in the region of Northeastern Argentina and Southern Paraguay. Int. J. Food Microbiol. 37, 231-235.

19. Windels, C.E.; Kommedahl, T. (1982). Pea cultivar effect on seed treatment with Penicillium oxalicum in the field. Ecological Epidemiology 72, 541-543.

20. Zhang, C.Q.; Liu, Y.H.; Ma, X.Y.; Feng, Z.; Ma, Z.H. (2009). Characterization of sensitivity of Rhizoctonia solani, causing rice sheath blight, to mepronil and boscalid. Crop Prot. 28, 381-386. 